\title{
Primer Simposio colombiano sobre métodos sico- físicos para el parto indoloro
}

Esta reunión científica organizada por el equipo de médicos de la Clínica Palermo de Bogotá (E. M. E. S. F. A. O.), se efectuó en el Salón de Conferencias de la Facultad de Medicina de la Universidad Javeriana, durante los días 4, 5, 6 y 7 de febrero.

En la ceremonia de instalación tomaron la palabra el señor Ministro de Salud, doctor Alejandro Jiménez Arango, el coordinador del EMESFAO, doctor Carlos R. Silva Mojica, el profesor Jorge Bejarano y el Rector de la Universidad Javeriana, reverendo padre Ortiz Restrepo, quienes hicieron resaltar la gran trascendencia del Simposio y la importancia del método sicoprofiláctico. En seguida el Equipo Médico de Estudios Sicofísicos: de Analgesia Obstétrica de Bogotá, D. E. obsequió un cocktail a los numerosos médicos inscritos y a los invitados entre los cuales se encontraban los presidentes de todas las sociedades científicas de Bogotá y destacadas personalidades del periodismo nacional.

Aportaron trabajos los siguientes equipos médicos:

Instituto Fernand Lamaze, de Cali.

Instituto de estudios sicofísicos para el parto. Cali.

Equipo de Sicoprofilaxis de la Clínica Los Angeles. Barranquilla.

Equipo de Sicoprofilaxis, de Medellín.

Equipo de Parto Indoloro, de Pereira.

Equipo de Sicoprofilaxis, de Pereira.

Grupo de Sicoprofilaxis, de Ibagué.

Equipo de Sicoprofilaxis de la Clínica de Marly.

Equipo de Sicoprofilaxis del Hospital San José, de Bogotá.

Centro número 9 del Distrito Especial de Bogotá.

Equipo Médico de Estudios Sicofísicos de Analgesia Obstétrica, de Bogotá, D. E.

Fue nombrado Presidente Honorario del Simposio el doctor Pierre Vellay, connotado científico en el campo de la sicoprofilaxis obstétrica mundial, quien llegará a Bogotá en la primera semana de marzo, a dictar un ciclo de conferencias en la Clínica Restrepo.

La lista de los asistentes es la siguiente:

BOGOTA.-Alvaro Medina Molina, Carlos Valencia, Gilberto Roldán, Germán Jordán, Esteban Pérez, Jaime Ramírez S., Francisco Luis García, Luis E. Guerrero Ramírez, Luis Torrado Núñez, Carlos Arturo Guevara, Luis Laverde Mercado, Efraín González, Germán Moya, Alvaro Espinosa, Carlos 
Pacheco, Alvaro Pachón Rojas, Hernando Rodríguez, Rodulfo Camero, Rafael Zornosa del Valle, Francisco Jaramillo Mora, Hugo García, Jaime Pedraza, Ricarảo Forero, Jaime Afanador, Enrique Hurtado S., Luis Angulo R., Jorge Villarreal Mejía, Jaime Danies, Gonzalo Lora, Guillermo Hernández, Hernando Ramírez, Inés Pastrana, Mary Villamil Vargas, Cecilia Puerto Quintana, Ernestina Angarita, Elvia Castañeda de Garzón, Gloria Navarro, Cecilia de Mojica, Cecilia de Naber, Esteban París, Miguel A. Fernández B., Belisario Calderón, Hernando Amaya, Guillermo López-Escobar, Carlos $\mathbf{R}$. Silva Mojica, Alberto Gómez Tamayo, Hernán Saavedra Bayer, Guillermo Lesmes.

ARMENIA.-Miguel Gómez González, Héctor Agudelo Z., Ernesto Ramírez Molina.

CALI.-Jaime Múnera Angel, Tomás Becerra, Henry Cajiao, Cira Olivera.

MEDELLIN.-Gustavo Isaza Mejía, Gustavo Isaza C., Leonídas Rodríguez R., Jorge Muñoz Duque, Eduardo Yepes, Jorge Hernández López, Jaime Botero, Alfonso Cano.

PAIPA.-Pablo E. Perico.

CUCUTA.-Hugo Quijao Morales, David Dario Porras, Santiago Uribe Franco, Hernando Gómez.

PEREIRA.-Abel Villegas, Hugo García Vélez, José Antonio Vásquez.

CENTRO SANTANDER.-Custodio Rueda.

IBAGUE.-Gregorio Tovar Castaño, Mario Bueno Barrios, Reinalỏo Orjuela Gómez.

BARRANQUILLA.-Fernando Navas Uribe.

VILLAVICENCIO.-Antonio Carvajal.

PASTO.-Fernando Mora España, Alfonso Ortiz S., Luis Ignacio Becerra. ZIPAQUIRA.-Guillermo López Villarraga.

VENEZUELA.-Bruno Burruni.

GIRARDOT.-Jorge Benincore.

PAZ DEL RIO.-Enrique Vargas, Alfonso Arrieta.

CONCLUSIONES DEL I SIMPOSIO SOBRE IMETODOS SICOFISICOS DE ANALGESIA OBSTETRICA REUNIDO DEL 4 AL 7 DE FEBRERO EN LA CIUDAD DE BOGOTA, D. E.

El I Simposio Colombiano sobre métodos Sicofísicos de Analgesia Obstétrica, aprobó al clausurarse sus sesiones el día 7 de febrero de 1959, las siguientes conclusiones:

I.-La práctica de la preparación sicoprofiláctica de la embarazada debe ser intensificada en el territorio nacional.

II.-Deben incluírse nociones del método sicoprofiláctico dentro de los programas de las facultades de medicina y enfermería.

III.-Abogar ante las directivas de clínicas y hospitales, a fin de que se hagan las modificaciones necesarias (personal de internos y de enfermería, local, etc.), para el buen funcionamiento del método sicoprofiláctico. 
IV.-Propender por la formación de enfermeras instructoras especializadas y facilitar el entrenamiento de ellas en los centros de estudio del método.

V.-Se aconseja la formación de equipos médicos para la correcta ejecución del método.

VI.-T'oda mujer se beneficia, estando embarazada, con el método sicoprofiláctico. Dentro de condiciones especiales del medio en que se inicie su aplicación, debe tenerse en cuenta la posibilidad de selección para que un fracaso eventual no vaya a influir sobre la difusión del método.

\section{CLASES TEORICAS}

VII.-Adaptar el número de clases teóricas al tipo de pacientes y al medio en que se actúa, recomendando un mínimo de seis, e incluyendo dentro de las primeras una para maridos.

VIII.-La iniciación de las clases teóricas debe ser entre el sexto y septimo mes.

\section{CLASES PRACTICAS}

IX.-Deben ser iniciadas simultáneamente con las clases teóricas.

$\mathrm{X}$ - El entrenamiento neuro-muscular consta fundamentalmente de: relajación, respiración y pujo como procesos cerebrales activos que deben practicarse hasta el final del embarazo.

XI.-Si bien los ejercicios gimnásticos, no son fundamento del método, constituyen un buen auxiliar de higiene prenatal.

XII.-Los grupos no deben excecier de 20 señoras para la parte teórica y 10 para la parte práctica, con una duración no mayor de 45 minutos.

\section{APOYO DEL TRABAJO}

XIII.-Es lo más aconsejable que las señoras sean atendidas por el grupo de médicos que las prepara.

XIV.-El apoyo permanente activo es indispensable desde el momento deI ingreso de la señora a la clínica.

XV.-Para posteriores comunicaciones, insístase en el estudio del con(icionamiento extemporáneo para el parto.

XVI.-El método sicoprofiláctico debe ser considerado como una analgesia de base que no excluye el uso de drogas necesarias. Debe evitarse el uso de drogas y/o dosis que inhiben la actividad nerviosa superior (tipo escopolamina).

\section{CLASIFICACION DE RESULTADOS}

XVII.-EI Simposio reconoce la dificultad de hacer cualquier evaluación basada en una sensación tan compleja y subjetiva como el dolor, pero en la necesidad de dar normas para la comparación de estadisticas recomienda la siguiente clasificación: 
Casos excelentes.-Parto a plena conciencia y sin dolor.

Casos buenos.-Dolor tolerable y lucidez perfecta durante todo el parto.

Casos regulares.-Percepción de dolor controlable, deseo de alguna analgesia, quejas, alguna inquietud, pero responde a las órdenes.

Casos malos.-(Fracasos). Dolor intolerable, señora inconsciente en el momento del parto.

Para la valoración de tales resultados deben tenerse en cuenta tanto el concepto de la señora como el del médico.

XVIII.-Fúndese la Sociedad Colombiana de Estudios Sicofísicos de Analgesia Obstétrica; y nómbrese para el efecto una comisión, a fin de que estudie la elaboración de estatutos y reglamento.

XIX.-Desígnase como sede del próximo Simposio, que deberá celebrarse en febrero de 1960, a la ciudad de Medellín y con sede subsiguiente en 1961 a la ciudad de Cali.

Firmada por los doctores Fernando Navas U. (Barranquilla), Hugo García Vélez (Pereira), Abel Villegas (Pereira), Gustavo Isaza Mejía (Medellín), Carlos R. Silva Mojica, Belisario Calderón, Guillermo López-Escobar, Hernando Amaya y Miguel A. Fernández Bastidas (Bogotá). 